\title{
Ray Systems in Granular Cratering
}

\author{
Tapan Sabuwala, ${ }^{1}$ Christian Butcher, ${ }^{2}$ Gustavo Gioia, ${ }^{1}$ and Pinaki Chakraborty ${ }^{2, *}$ \\ ${ }^{1}$ Continuum Physics Unit, Okinawa Institute of Science and Technology Graduate University, Onna-son, Okinawa 904-0495, Japan \\ ${ }^{2}$ Fluid Mechanics Unit, Okinawa Institute of Science and Technology Graduate University, Onna-son, Okinawa 904-0495, Japan
}

(Received 18 February 2018; published 27 June 2018)

\begin{abstract}
In classical experiments of granular cratering, a ball dropped on an evened-out bed of grains ends up within a crater surrounded by a uniform blanket of ejecta. In this Letter, we show that the uniform blanket of ejecta changes to a ray system, or set of radial streaks of ejecta, where the surface of the granular bed includes undulations, a factor that has not been addressed to date. By carrying out numerous experiments and computational simulations thereof, we ascertain that the number of rays in a ray system $\propto D / \lambda$, where $D$ is the diameter of the ball and $\lambda$ is the wavelength of the undulations. Further, we show that the ejecta in a ray system originates in a narrow annulus of diameter $D$ with the center at the site of impact. Our findings may help shed light on the enigmatic ray systems that ring many impact craters on the Moon and other planetary bodies.
\end{abstract}

DOI: $10.1103 /$ PhysRevLett.120.264501

Ray systems [1] became known shortly after the advent of the telescope, perhaps in 1647, when Johannes Hevelius published what might have been the first map of the Moon to show them. Although they were recognized as settled ejecta, that is, deposits of debris thrown out when a meteorite impacts the surface of a planetary body, their origin proved to be the sort of question for which competing theories abound to date [2-4]. Indeed, Jules Verne betrayed no ignorance when, in reference to a ray system on the Moon, he asked, "By what geological phenomena could this blazing coma have been possibly produced?" [5].

Motivated by observations of ray systems in planetary cratering, we study an analog system: granular cratering [6,7]. This simple analog-a ball impacting on a granular bed-has been widely used to study the characteristics of planetary craters [8-11] and, more relevant to ray systems, the dynamics of ejecta [4,11-18]. From low-velocity impacts (meaning, impact velocity $<c$, the speed of sound in the granular bed; typically $c \sim O(100) \mathrm{m} / \mathrm{s}$ [19]) to hypervelocity impacts (meaning, impact velocity $>c$ ) typical of planetary cratering, experiments and simulations show that the ejecta dynamics remain remarkably invariant. For instance, across the range of impact velocities, the ejecta sweeping outward from the impacting ball as a conical curtain is launched at a near-constant angle of $\approx 50^{\circ}$ with the bed surface, its velocity decaying selfsimilarly as a power law of the distance from impact

Published by the American Physical Society under the terms of the Creative Commons Attribution 4.0 International license. Further distribution of this work must maintain attribution to the author(s) and the published article's title, journal citation, and DOI.
$[11,12,14,16-18,20]$. In short, in order to investigate the dynamics of ejecta, granular cratering may be deemed a suitable analog of planetary cratering.

Although granular cratering has captured many features of planetary craters, ray systems have persistently remained outside its purview. In a typical low-velocity experiment, a vessel full of grains (of diameter $d \sim 50 \mu \mathrm{m}$ ) is shaken to form a homogeneous bed, the surface of which is subsequently evened out by scraping off the top. When a ball (of diameter $D \sim 500 d$ ) is dropped on the bed from a height of about $1 \mathrm{~m}$, it forms a crater by ejecting the grains, which settle around the crater as a nearly uniform ejecta blanket without a ray system (Fig. 1(a); Supplemental Video 1 [21]).

To proceed, we draw inspiration from an unusual source. In noticeable contrast to the experiments reported in the scientific literature, similar experiments by school students often show hints of ray systems (see Ref. [21]). By contrasting the experimental protocols, we infer that this discrepancy stems from a subtle factor-in the school experiments, the surface of the granular bed is not evened out before dropping the ball. Shaped by the pouring of the grains, the surface stays undulating, akin to planetary surfaces [26]. Remarkably, when we conduct experiments with an undulating surface [of wavelength about 50d; see inset of Fig. 1(b)], the ejecta settles on an unambiguous ray system (Fig. 1(b); Supplemental Video 2 [21]). The undulating surface shapes the ejecta to form the apparition of a ray system.

To elucidate the role of the undulations, we consider granular beds with regular surface undulations. We create such a surface by pressing a hexagonal grid of wavelength $\lambda$ onto a granular bed that had been evened out by scraping. (For regular surface undulations of other shapes, see Ref. [21].) Spanning a range of $\lambda, D$, the impact velocity of the ball, the size of the granular bed, the packing fraction 


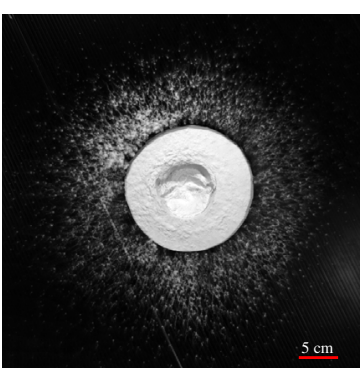

(a)

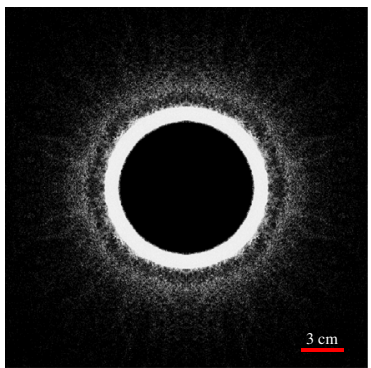

(e)

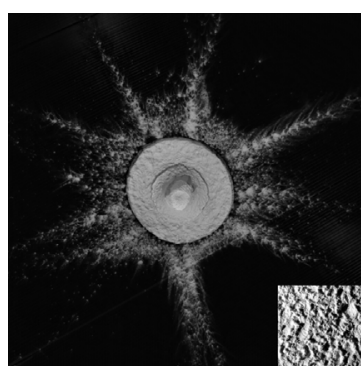

(b)

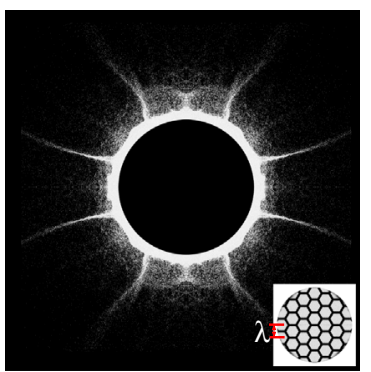

(f)

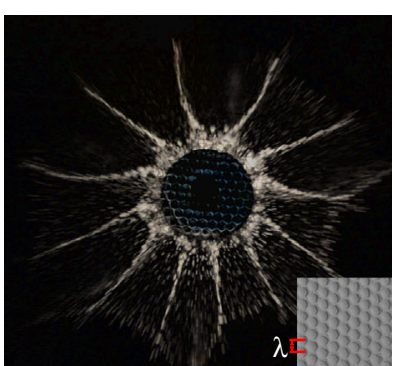

(c)

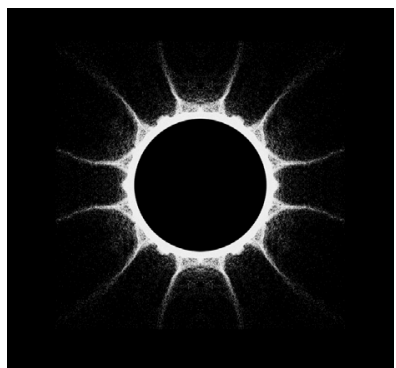

(g)

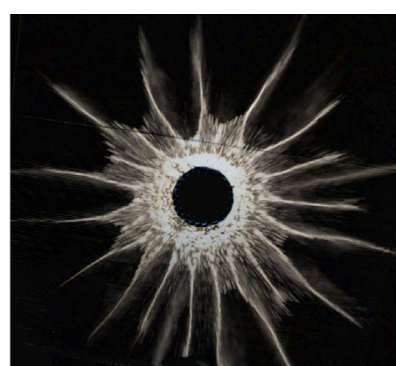

(d)

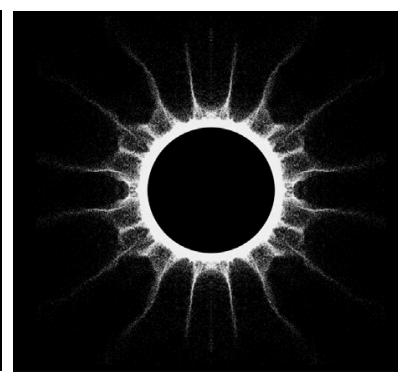

(h)

FIG. 1. Top view of ejecta in low-velocity experiments (a)-(d) and hypervelocity simulations (e)-(h). (a) Ejecta blanket in a typical experiment with an evened-out surface. Note the absence of a ray system. (b) Ejecta blanket in an experiment with an undulating surface (see inset). Note the ray system. (c),(d) Ejecta curtains showing ray systems in experiments with hexagonal surface undulations of wavelength $\lambda$ [see inset of panel (c)]; $D / \lambda=2.93$ (c) and $5.86(\mathrm{~d})$. (e) Ejecta curtain showing the absence of a ray system in a typical simulation with an evened-out surface. (f)-(h) Ejecta curtains showing ray systems in simulations with hexagonal surface undulations of wavelength $\lambda$ [see inset of panel (f)]; $D / \lambda=2.23$ (f), 3.45 (g), and 4.75 (h). Impact velocity $=20 \mathrm{~m} / \mathrm{s}$ for the simulations shown here. In all simulations, we simulate one quadrant and mirror the ejecta pattern assuming symmetry. In panels (d) and (h), in addition to the prominent rays, we can also see secondary rays (see Ref. [21]).

of the granular bed, and the ambient humidity, we carry out 135 experiments in total (see Ref. [21]). Each experiment manifests an unambiguous ray system (Figs. 1(c) and 1(d); Supplemental Video 3 [21]). We focus on $N$, the number of prominent rays (meaning, the longest and most conspicuous rays) in a ray system and find that $N$ depends only on $D$ and $\lambda$. Invoking dimensional analysis, we note that $N$ (which is unitless) can only depend on the unitless ratio $D / \lambda$. Indeed, our experiments substantiate this constraint and further show that $N$ varies linearly with $D / \lambda$ (Fig. 2).

For a closer look at the mechanism underlying ray systems, we turn to computational simulations. To this end, we use the discrete-element method in which each grain is represented by a sphere endowed with mass (a "discrete element"); the grain interacts with other grains through contact forces and its motion is governed by Newton's laws. We conduct simulations for impact velocities that span the range from low-velocity to hypervelocity impacts [27] and for various values of $D / \lambda$. Echoing the results from the low-velocity experiments, each simulation with an undulating surface yields an unambiguous ray system [Figs. 1(f)-1(h)], whereas for an evened-out surface, the ray system disappears [Fig. 1(e)] [28].

Each impact generates a shock wave in the granular bed [Fig. 3(a)]—a weak shock wave for a low-velocity impact and a strong shock wave for a hypervelocity impact. On reaching the bed surface, the (compressive) shock wave reflects back as a rarefaction wave. These waves induce a flow field that ejects the surficial grains. The flow field includes a surface-normal velocity component-this plays a key role in forming rays. For an evened-out surface, the

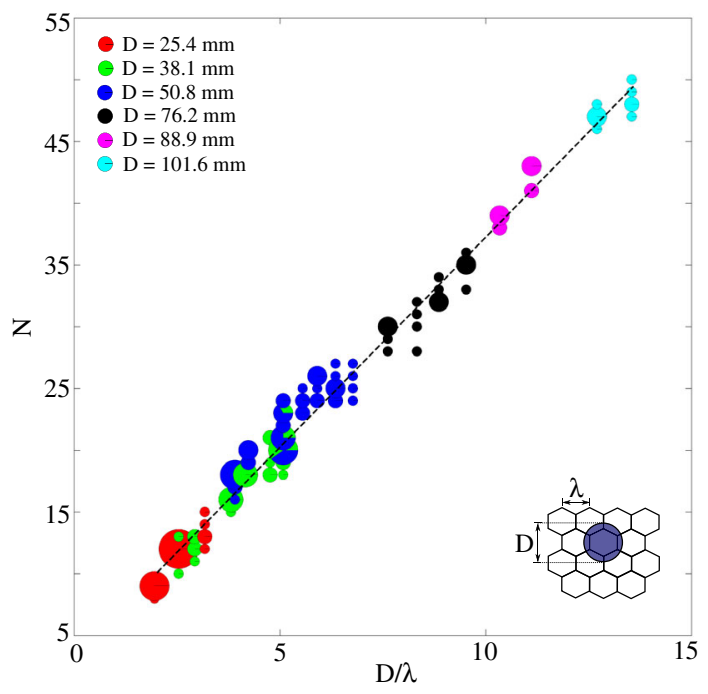

FIG. 2. $\quad N$ vs $D / \lambda$. Data from 135 low-velocity experiments with hexagonal surface undulations (see inset; blue circle denotes the impacting ball). The size of the data points (circles) is proportional to the number of overlapping points, with the area corresponding to any color proportional to the attendant value of $D$. The dashed line shows the best fit using linear regression $\left(R^{2}=0.98\right)$. 


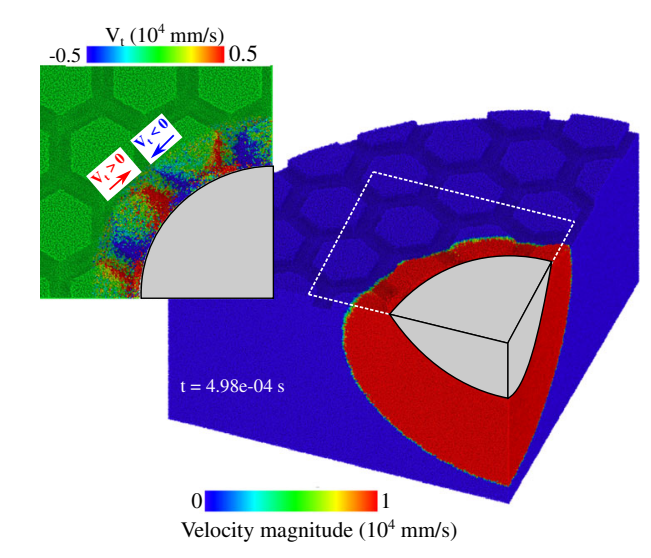

(a)

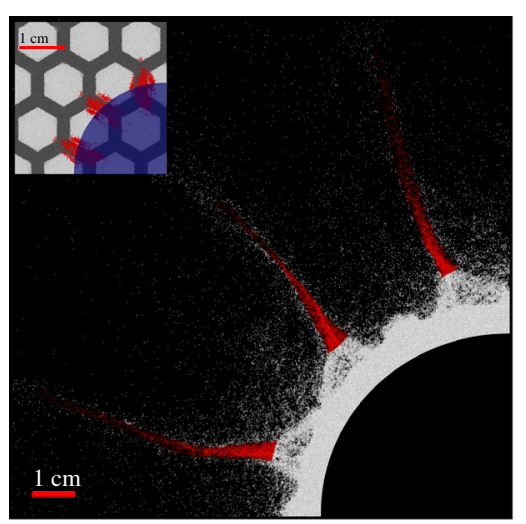

(b)

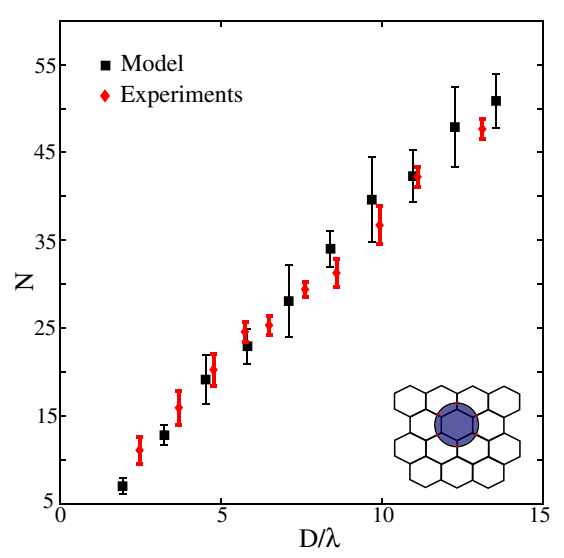

(c)

FIG. 3. Origin of ray systems. (a) A snapshot of grain velocities in the granular bed. At time $t=0$, the ball impacts the bed surface with velocity $=20 \mathrm{~m} / \mathrm{s}$. We show the simulated quadrant of the granular bed in the undeformed configuration; $D / \lambda=3.45$ for the hexagonal surface undulations. The gray region marks the current position of the ball inside the granular bed. The sharp interface between moving and stationary grains marks the shock wave. Inset: Azimuthal velocity $V_{t}$ on the surface patch demarcated by dotted lines. Induced by the surface-normal velocity from the valley sidewalls, $V_{t}$ focuses the affected grains into the radially outbound ejecta, thereby engendering a ray system. (b) Top view of the ray system produced by the impact shown in panel (a) [cf. Fig. 1(g)]. The grains in the rays are marked in red. Inset: Enlarged view of the preimpact surface. The ray-forming grains (red grains) straddle the edge of the impacting ball (blue quarter circle). (c) $N$ vs $D / \lambda$. We show experimental data of Fig. 2 (red) and predictions from the geometric model (black), both binned with a bin size $\delta(D / \lambda)=1$. The vertical bars span 2 standard deviations of $N$. In the geometric model, for simplicity, we ignore the thickness of the valleys and idealize them as one-dimensional lines (see inset). Counting the number of intersections between the valleys and the edge of the ball (blue circle) yields the prediction for $N$.

flow field is axisymmetric, and the ejecta settles on an axisymmetric, uniform blanket. By contrast, for an undulating surface, the surface-normal velocity from the sidewalls of valleys focuses the ejecta into a ray system [inset of Fig. 3(a)]. Reminiscent of the Munroe effect in shaped charges [29], such a focused ejection of rays has also been reported in fluids with an impulsively accelerated concave surface [30]. We further note that because shock waves (and attendant flow fields) are generated in low-velocity as well as hypervelocity impacts, ray systems are engendered across the range of impact velocities, with $N$ invariant to the impact velocity — as is indeed seen in our simulations.

For further insight into ray systems, we follow the evolution of the ejecta and pick an instant when the rays are clearly visible. We mark the grains in the rays and trace them backward in time [Fig. 3(b)]. Ejected at early stages of the impact, these grains come from a narrow annulus of valleys that straddles the edge of the impacting ball [inset of Fig. 3(b)]. Each valley engenders a distinct ray. This finding affords a simple geometric model: $N$ is set by the number of valleys intersected by the edge of the ball. That is, we count the number of intersections between a circle of diameter $D$ (representing the ball) and the arms of the hexagonal grid of wavelength $\lambda$ (representing the valleys). In counting the number of intersections, we vary the center of the circle over the spatial extent of a hexagon because the ball in the experiments can land anywhere over this region. Consequently, for a fixed $D / \lambda$, we get a small variation in the predicted $N$. The predictions from this geometric model over the entire span of $D / \lambda$ are in excellent accord with the experiments [Fig. 3(c)].
The geometric model also allows us to address an inverse problem: what was the diameter of the impactor that formed a given crater? Of central importance in planetary cratering, this problem poses a formidable challenge. The crater diameter depends on many factors [31] - the diameter, velocity, and density of the impactor; the density, strength, and porosity of the planetary crust; the surface gravity, among others. These parameters are typically illconstrained for planetary bodies, thereby leading to large uncertainties in the estimated impactor diameter. For example, for a crater the size of Barringer Crater (Arizona), depending on the values of the parameters, the impactor diameter estimated from scaling laws varies by $400 \%$ [32]. To complement and constrain such estimates, next we discuss a novel and simple tool.

Per the geometric model, $N$ is set by the number of valleys straddling the edge of the impactor. Thus, given $N$, we can estimate the impactor diameter $D$ as the diameter of the circle that intersects $N$ valleys in the preimpact surface. To test this tool, we conduct experiments on granular beds with irregular surface undulations (Fig. 4(a); Ref. [21]), analogous to planetary surfaces. These experiments reveal that only the prominent valleys - meaning, valleys that are deep relative to their surroundings - at the edge of the impactor produce prominent rays (see Ref. [21]). To estimate $D$, we analyze a patch of the preimpact surface [Fig. 4(b)]. From this patch, we extract one-dimensional topographic profiles along concentric circles centered at the point of impact. For each profile, which corresponds to a circle of diameter $D_{c}$, we count the number of prominent valleys $N_{v}$ (see Ref. [21]). 


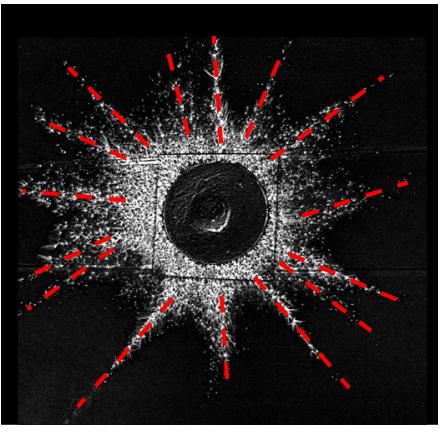

(a)

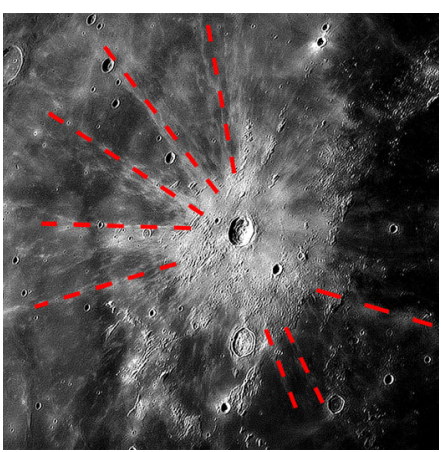

(d)

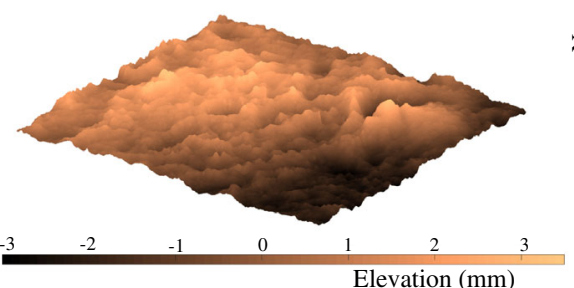

(b)

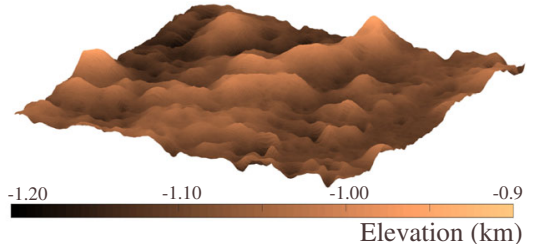

(e)

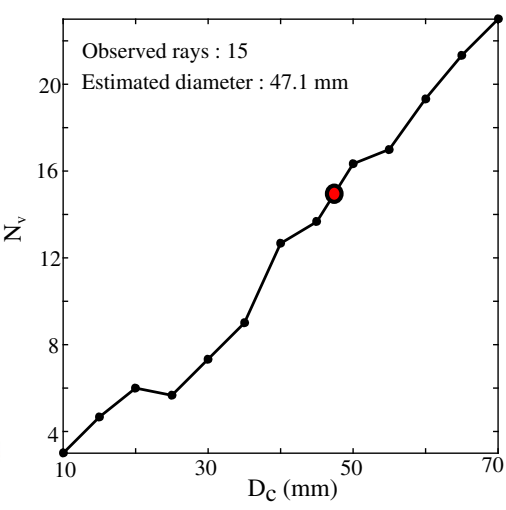

(c)

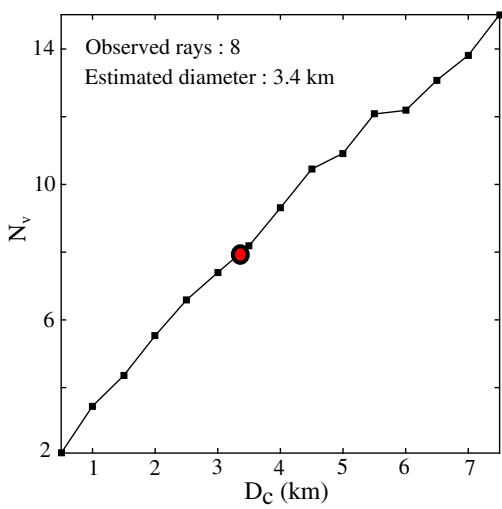

(f)

FIG. 4. Estimating impactor diameter: a granular crater (a)-(c) and the lunar crater Kepler (d)-(f). (a),(d) Ray systems in the ejecta blanket. The prominent rays are marked by dashed red lines. The granular ray system is produced by dropping a 50.8-mmdiameter steel ball on a granular bed with irregular surface undulations. (b),(e) Preimpact surfaces. For the granular crater, we obtain the terrain map of the preimpact surface using a laser scanner (David-SLS-2); the patch size is $10 \times 10 \mathrm{~cm}^{2}$. For Kepler, we obtain the terrain data from the SLDEM 2015 database [33]; the patch size is $10 \times 10 \mathrm{~km}^{2}$. (e),(f) $N_{v}$ vs $D_{c}$. For Kepler, this plot is obtained by averaging the $N_{v}$ vs $D_{c}$ data from 75 terrain patches. The estimated impactor diameter $D$ is the $D_{c}$ corresponding to $N_{v}=N$ - these points are marked by red dots.

From a plot of $N_{v}$ vs $D_{c}$, we can readily estimate the impactor diameter $D$ as the $D_{c}$ that corresponds to $N_{v}=N$ [Fig. 4(c)].

Extrapolating this tool to lunar craters, we consider the crater Kepler. (For the crater Tycho, see Ref. [21].) We visually identify eight prominent rays, in accord with previous observations [34] [Fig. 4(d)]. Because the preimpact surface has long been obliterated by the impact, as a proxy for the preimpact surface, we analyze 75 azimuthally distributed patches of lunar terrain from the annular region located within the radii of 1.5 and 2 crater diameters from the center of the crater [Fig. 4(e)]. For each patch, we extract onedimensional topographic profiles along concentric circles centered at the centroid of the patch. Following the procedure outlined above, we estimate $D=3.4 \mathrm{~km}$, comparable with the estimate of $2.5 \mathrm{~km}$ from scaling laws [Fig. 4(f)].

In summary, using experiments and simulations, we have shown that ray systems are but a generic feature of impact on undulating surfaces. Focusing on a single characteristic of ray systems - the number of prominent rays-we have proposed a simple tool to estimate the diameter of the impactor. We posit that other characteristics of ray systems also inherit a signature of the impact. For example, analyzing the length of the rays may help constrain the energy of the impact. Further, studying the role of the shape and orientation of the valleys may unveil additional features of ray systems. While much still remains hidden, the long elusive ray systems are beginning to yield their secrets.

We thank Sue Kieffer, Jay Melosh, Troy Shinbrot, and William Powell for discussions. This work was supported by the Okinawa Institute of Science and Technology Graduate University.

*pinaki@oist.jp

[1] H.J. Melosh, Impact Cratering: A Geologic Process, Oxford Monographs on Geology and Geophysics Vol. 11 (Oxford University Press, New York, 1989).

[2] B. R. Hawke, D. Blewett, P. Lucey, G. Smith, J. Bell, B. Campbell, and M. Robinson, Icarus 170, 1 (2004).

[3] V. Shuvalov, Meteorit. Planet. Sci. 47, 262 (2012). 
[4] T. Kadono, A. Suzuki, K. Wada, N. Mitani, S. Yamamoto, M. Arakawa, S. Sugita, J. Haruyama, and A. Nakamura, Icarus 250, 215 (2015).

[5] J. Verne, Autour de la lune (J. Hetzel, Paris, 1872) [English translation by E. Roth, All Around the Moon (King \& Baird, Philadelphia, 1876)].

[6] H. Katsuragi, Physics of Soft Impact and Cratering (Springer, New York, 2016).

[7] J. Ruiz-Suárez, Rep. Prog. Phys. 76, 066601 (2013).

[8] A. M. Walsh, K. E. Holloway, P. Habdas, and J. R. de Bruyn, Phys. Rev. Lett. 91, 104301 (2003).

[9] J. S. Uehara, M. A. Ambroso, R. P. Ojha, and D. J. Durian, Phys. Rev. Lett. 90, 194301 (2003).

[10] S. Yamamoto, K. Wada, N. Okabe, and T. Matsui, Icarus 183, 215 (2006).

[11] K. Wada, H. Senshu, and T. Matsui, Icarus 180, 528 (2006).

[12] J. Marston, E. Li, and S. Thoroddsen, J. Fluid Mech. 704, 5 (2012).

[13] J. F. Boudet, Y. Amarouchene, and H. Kellay, Phys. Rev. Lett. 96, 158001 (2006).

[14] S. Deboeuf, P. Gondret, and M. Rabaud, Phys. Rev. E 79, 041306 (2009).

[15] D. Stöffler, D. Gault, J. Wedekind, and G. Polkowski, J. Geophys. Res. 80, 4062 (1975).

[16] M. J. Cintala and R. A. Grieve, Meteorit. Planet. Sci. 33, 889 (1998).

[17] B. Hermalyn and P. H. Schultz, Icarus 216, 269 (2011).

[18] S. Tsujido, M. Arakawa, A. Suzuki, and M. Yasui, in Asteroids, Comets, Meteors 2014 (2014), http://adsabs .harvard.edu/abs/2014acm..conf..537T.

[19] C.-h. Liu and S. R. Nagel, Phys. Rev. B 48, 15646 (1993).

[20] K. Wünnemann, M.-H. Zhu, and D. Stöffler, Meteorit. Planet. Sci. 51, 1762 (2016).

[21] See Supplemental Material at http://link.aps.org/ supplemental/10.1103/PhysRevLett.120.264501 for additional discussion, which includes Refs. [22-25], and for Supplemental Videos (SV1-SV3).
[22] C. Kloss, C. Goniva, A. Hager, S. Amberger, and S. Pirker, Prog. Comput. Fluid Dyn. 12, 140 (2012).

[23] R. M. Schmidt and K. R. Housen, Int. J. Impact Eng. 5, 543 (1987).

[24] S. K. Croft, J. Geophys. Res. Solid Earth 90, C828 (1985).

[25] Z. Yue, B. Johnson, D. Minton, H. Melosh, K. Di, W. Hu, and Y. Liu, Nat. Geosci. 6, 435 (2013).

[26] Such an undulating surface mimics a planetary surface more closely than a smooth surface with an isolated, large, hemispherical preexisting crater, as has been previously studied using hydrocode simulations of planetary cratering [3].

[27] We vary the impact velocities from 4 to $40 \mathrm{~m} / \mathrm{s}$. To compare with $c$, we first note that the value of $c$ depends on the distance from the surface of the bed. As reference, we take this distance to be $D / 2$, where the edge of the impacting ball meets the bed surface. For our simulations, $c=16.9 / \mathrm{s}$, wherein we have chosen the material parameters so that the value of $c$ is an order of magnitude lower than what is typical for granular materials (see Ref. [21]).

[28] Additionally, note that unlike the experiments, our simulations lack ambient air. Yet, both experiments and simulations yield ray systems signifying that the formation of ray systems is indifferent to ambient air.

[29] C. E. Munroe, Popular Science Monthly 56, 444 (1900).

[30] A. Kiyama, Y. Tagawa, K. Ando, and M. Kameda, J. Fluid Mech. 787, 224 (2016).

[31] K. R. Housen and K. A. Holsapple, Icarus 211, 856 (2011).

[32] K. Wünnemann, D. Nowka, G. Collins, D. Elbeshausen, and M. Bierhaus, in Proceedings of the 11th Hypervelocity Impact Symposium, edited by F. Schäfer and S. Hiermaier (Fraunhofer-Verlag, Germany, 2011), pp. 1-16.

[33] M. Barker, E. Mazarico, G. Neumann, M. Zuber, J. Haruyama, and D. Smith, Icarus 273, 346 (2016).

[34] J. Van Diggelen, The Moon 1, 67 (1969). 